\title{
The 2nd Annual Shanghai Symposium on Chemical and Pharmaceutical Structure Analysis
}

\author{
The Changing Paradigm in Drug Discovery and Development: East Meets West \\ Shanghai, China, 13-16 April 201I
}

The 2nd Annual Shanghai Symposium on Chemical and Pharmaceutical Structure Analysis (CPSA Shanghai 20II) was held on 13-16 April 20II in Shanghai, China. The CPSA Shanghai meetings began in 2010 and are an international extension of the CPSA USA annual meetings - the first industry-led event to focus on the specific needs of industry researchers. The goal of the CPSA meetings is to provide an in-depth review of innovative technology and industry practices through open discussion of industry-related issues and needs. Education and specialized training are the foundation of all CPSA events: 'Where technology and solutions meet'.

Parallel to its fast economical growth, China is also among the fastest growing countries in pharmaceutical research and development. Thus, the goal of the 2nd Annual Shanghai Symposium on Chemical and Pharmaceutical Structure Analysis (CPSA Shanghai 2011) is to bring together the scientists from the East and the West to provide a unique, in-depth review of innovative technology and industry practices through open discussions of industry-related issues and needs. Of course, another goal of CPSA Shanghai is to promote valuable cultural exchanges: 'Where East meets West'.

Jing-Tao Wu of Millennium Pharmaceuticals served as CPSA Shanghai 2011 Program Chair. The theme of the meeting 'Changing paradigm of drug discovery: East meets West' reflects a new trend in the globalization of the pharmaceutical research and development environment. This globalization starts with offshore outsourcing, integrated research and development capability, and leads to global R\&D, registration and marketing strategies. To open the conference, the Program Chair welcomed the participants and summarized the three main threads of the symposium: absorption, distribution, metabolism and excretion (ADME)/bioanalysis; pharmaceutical sciences/analysis; and translational medicine/analytical strategy. The close connection of analytical sciences and the disciplines they support, each represented by leaders in the respective field was a unique feature of this symposium.
Pete Smith, Senior Vice President of Nonclinical Development Sciences at Millennium Pharmaceuticals delivered the Plenary Lecture. His talk on 'Challenges and future direction of oncology drug development' provided a unique perspective on the current and future direction of the pharmaceutical industry. A shift in the US FDA requirements and an increasingly segmented patient population was delineated. To meet these challenges, the drugdevelopment paradigm in oncology is changing from drug- to patient-centric, from target- to pathway-focused and from single agent to combinations. Translational medicine and biomarkers really hold the key for future success in the oncology area.

Absorption, distribution, metabolism and excretion/bioanalysis is the main topic of the symposium. The ADME sessions started with a comparison of regulatory requirement on ADME package among China, the USA and Europe. Dafang Zhong of Shanghai Institute of Materia Medica kicked off the discussion by giving an overview of the drug metabolism and pharmacokinetics regulatory package in China. Mingshe Zhu of Bristol-Myers Squibb and Lawrence Gan of Biogen Idec went on to deliver two excellent presentations that highlighted the spirit of two ADME-related guidances on metabolite in safety testing and drug-drug interactions. In general, the ADMErelated regulatory package has not been fully
Mike S Lee ${ }^{\dagger l}$ \& Jing-Tao $\mathbf{W u}^{2}$

${ }^{\dagger}$ Author for correspondence: 'Milestone Development Services, PO Box 178, Newtown, PA 18940-0178, USA

2Millennium Pharmaceuticals Inc.

Cambridge, MA 02139, USA

Tel.: +l 2677570462

Fax: + I 2677570463

E-mail: info@

milestonedevelopment.com 
developed in China compared with the west but China also has some specific requirements. The ADME sessions went on with specialized topics in drug-drug interactions, biologics development and pharmacokinetics/pharmacodynamics. The topic of drug-drug interaction with herbal medicines presented by Yan Sun of Shanghai Institute of Materia Medica and the topic of biologics drug-drug interactions, presented by Lewis Klunk of Biogen Idec generated great discussions on these hot topics. Another highlight of the ADME sessions was when four industry veterans presented real world experience on using ADME to advance compounds in drug discovery and development. The case example given by Vic Kadambi of Millennium Pharmaceuticals further extended the ADME optimization into predictive toxicology.

The bioanalytical sessions started with a discussion of emerging bioanalytical technologies organized by Naidong Weng of Johnson \& Johnson. Tom Verhaeghe of Johnson \& Johnson and Luke Bi of Covance presented excellent case examples of the use of dried blood spot sampling in clinical bioanalysis and nonclinical toxicokinetic studies, respectively. The emerging issues and needs in regulated bioanalysis were openly discussed in the first 'Regulated Bioanalysis Summit'. The Summit featured two parts. Part I on 'Regulated bioanalysis: state-ofthe-art' was chaired by Danlin Wu of Roche. One key issue raised during the session was the need for harmonization at a global level. Mark Arnold of Bristol-Myers Squibb addressed the impact of regional differences on reports and filings and also provided an update on activities of the Global Bioanalysis Consortium and steps toward harmonized regulations. A CRO perspective was shared by Jinsong Xing of WuXi AppTec, as recent strategies for development and validation were discussed. The emerging issues and needs for ligand-binding assays were highlighted in the session. Roland Staak of Roche focused on the importance of reagent quality for ligand-binding assays. Recent experiences and insights on the use of ligand-binding assays for protein therapeutics and immunogenicty testing was provided by Eginhard Schick of Roche. Part II of the Regulated Bioanalysis Summit featured a session on 'Current issues in regulated bioanalysis' chaired by Anne Aubry of Bristol-Myers Squibb. Aubry led the session with real world experiences with unexpected event investigations. The theme of a global-international bioanalytical laboratory continued with perspectives on bioanalytical method transfer given by Naidong Weng of Johnson \& Johnson. Indeed, outsourcing activities within bioanalytical laboratories has become the norm and as a result, method transfer to multiple sites has dramatically increased. XinPing Fang of XenoBiotic Laboratories concluded with some perspectives on harmonization experiences in laboratories in both the USA and China.

The pharmaceutical sciences/analysis track was the second main thread of the symposium. It compromised of a session on emerging trends and technology, a session on regulatory requirement and a pharmaceutical analysis summit. The summit gathered six highly regarded analytical scientists with two focused topics: highspeed, high-resolution separations and low-level impurity quantitation.

The third main thread of the symposium was biomarkers and their analytical strategy. Scott Fountain of Pfizer kicked off the session with a comprehensive review of the use of target and mechanism biomarkers in drug discovery and development. LiBin Ma of Covance then provided a CRO perspective on implementing biomarkers in clinical trials on a global scale. The session closed with an excellent case example of using a proteomic approach to the development of plasma biomarkers for predicting pre-term birth presented by Kevin Rosenblatt of the Brown Foundation Institute of Molecular Medicine.

At CPSA Shanghai 2011, for the first time, the Young Scientist Excellence Award was introduced. This exciting and unique event was held in conjunction with the Poster Session. The award recognizes the rising stars in a pharmaceutical-related discipline to encourage and promote young scientists to pursue a career in our industry. The poster session and the CPSA Shanghai Young Scientist Excellence Award ceremony were highlights of the meeting.

A combination of premier science and a memorable social event was provided at the Keynote Dinner Lectures hosted and sponsored by Agilent Technologies. John Pouk, Vice President and General Manager of Agilent Technologies delivered a celebration toast to kick-off the evening event followed by two Keynote Lectures and dinner. Lester Taylor of Agilent Technologies gave the attendees a provocative look into future technologies dedicated to 'Qualitative/quantitative applications' with the simultaneous determination of pharmacokinetic and metabolite profile using a highresolution quadrupole time-of-flight (QTOF) instrument. The implications that metabolites 
can potentially have an effect on safety and efficacy were delineated in the Keynote Lecture delivered by Scott Obach of Pfizer. As Scott pointed out, although the FDA recently issued guidance regarding safety testing on circulating chemically stable metabolites, examples of circulating chemically stable metabolites causing a toxicity unrelated to effects already possessed by the parent compound are unknown. While in vitro assays can be predictive in assessing the reactivity of metabolites, Scott warned the audience, such assays results, when considered alone, can be a poor indicator of the potential for toxicity.

The sponsored workshops provided a unique opportunity for detailed discussion (mostly in Chinese) on emerging technologies and services. The workshop sessions on the Wednesday featured hot topics in the field of biotransformation, bioanalysis and pharmaceutical analysis. Other workshops featured topics on: high-resolution MS for bioanalysis; equipment leasing; quantitation of peptides and proteins; identification of impurities; ultra-low flow nanospray applications; plasma sample clean-up; and human pharmacokinetic dose prediction.

A joint session with local bioanalytical and drug-metabolism discussion groups was held at the Shanghai Institute of Materia Medica. Jeff Zhang of Novartis provided an updated overview and perspective on the drug metabolism and pharmacokinetics process in drug discovery and development. Li Yu of Hoffmann-La Roche discussed recent applications of pharmacokinetics/pharmacodynamics that highlighted both key fundamentals and industry trends. The joint session closed with a lecture by Zhe-ming Gu of Xenobiotic Laboratories who provided expert insights into the application of radioisotopes for ADME studies.
Plans are already underway for the 3rd Annual Shanghai Symposium on Chemical and Pharmaceutical Structure Analysis (CPSA Shanghai 2012). The meeting will be held between 25-27 April 2012 at the Renaissance Shanghai Pudong Hotel. Jenny Zheng of Pfizer will serve as CPSA Shanghai 2012 Program Chair. More details on the program and events will be announced soon.

For more background information and details regarding the annual CPSA Shanghai meetings, please visit the conference website [1] or contact Mike Lee at info@milestonedevelopment.com.

\section{Acknowledgements}

The authors thank the Young Scientist Excellence Award Committee for their vision and leadership.

The authors also acknowledge Jianguo An - Wilmington PharmaTech, China; Zack Cheng (Co-Chair) GlaxoSmithKline, China; Lawrence Gan - Biogen-Idec, USA; Xiaoyi Gong - Merck, USA; Wenfang Miao (Co-Chair) - Pharmaron, China; Danlin Wu-Roche, USA; Su Zeng - University of Zhengjiang, China; Xiangmin Zhang - Fudan University, China; Mingshe Zhu (Coordinator) - Bristol-Myers Squibb, USA.

Financial \& competing interests disclosure

The authors have no relevant affliations or financial involvement with any organization or entity with a financial interest in or financial conflict with the subject matter or materials discussed in the manuscript. This includes employment, consultancies, honoraria, stock ownership or options, expert testimony, grants or patents received or pending, or royalties.

No writing assistance was utilized in the production of this manuscript.

\section{Website}

1 Annual Shanghai Symposium on Chemical and Pharmaceutical Structure Analysis. www.cpsa-shanghai.com 